\title{
Mitochondrial changes and oxidative stress in a mouse model of Zellweger syndrome neuropathogenesis
}

Rani Sadia Rahim ${ }^{\mathrm{a}}$, Mo Chen ${ }^{\mathrm{a}}$, C. Cathrin Nourse ${ }^{\mathrm{a}}$, Adrian C.B. Meedeniya ${ }^{\mathrm{b}}$, and Denis I Crane $^{a_{1} *}$

${ }^{\text {a}}$ Eskitis Institute for Drug Discovery, and School of Natural Sciences, Griffith University, Qld,

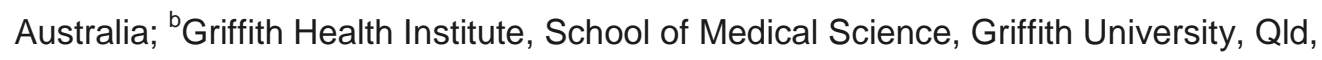
Australia.

${ }^{*}$ Corresponding author. Address: School of Natural Sciences, Griffith University, Nathan, Qld 4111, Australia. Tel: (617)37357253; fax: (617)37357773. Email address:

d.crane@griffith.edu.au.

Key words: Zellweger syndrome; PEX13 deficiency; Mitochondria; Oxidative stress; Peroxisomes.

Abbreviations: IF, immunofluorescence; TPH2, tryptophan hydroxylase-2; ZS, Zellweger syndrome; 


\section{ABSTRACT}

Zellweger syndrome (ZS) is a peroxisome biogenesis disorder that involves significant neuropathology, the molecular basis of which is still poorly understood. Using a mouse model of ZS with brain-restricted deficiency of the peroxisome biogenesis protein PEX13, we demonstrated an expanded and morphologically modified brain mitochondrial population. Cultured fibroblasts from PEX13-deficient mouse embryo displayed similar changes, as well as increased levels of mitochondrial superoxide and membrane depolarization; this phenotype was rescued by antioxidant treatment. Significant oxidative damage to neurons in brain was indicated by products of lipid and DNA oxidation. Similar overall changes were observed for glial cells. In toto, these findings suggest that mitochondrial oxidative stress and aberrant mitochondrial dynamics are associated with the neuropathology arising from PEX13 deficiency. 


\section{Introduction}

Zellweger syndrome (ZS) is a neonatal lethal peroxisome biogenesis disorder that arises from mutations in PEX genes and which is characterized by peroxisomal metabolic dysfunction (Distel et al., 1996, Gould and Valle, 2000).

Our understanding of the neuropathogenesis of ZS has been assisted by investigations on mouse models of ZS that have been generated by knockout or conditional inactivation of the PEX2 (Faust and Hatten, 1997, Faust, 2003), PEX5 (Baes et al., 1997, Janssen et al., 2000, Kassmann et al., 2007, Krysko et al., 2007, Hulshagen et al., 2008, Bottelbergs et al., 2010, Bottelbergs et al., 2012) and PEX13 genes (Maxwell et al., 2003, Muller et al., 2011). Our investigations have focused on PEX13 mutants. PEX13 is a component of the peroxisome matrix protein import machinery (Elgersma et al., 1996, Erdmann and Blobel, 1996, Gould et al., 1996) and PEX13 mutations in humans lead to disease across the Zellweger phenotypic spectrum (Liu et al., 1999, Shimozawa et al., 1999). PEX13 knockout mice model many of the disease phenotype of ZS patients, such as neonatal lethality, abnormal peroxisome metabolism, broad tissue pathology, a neuronal migration defect, and brain dysmorphology (Maxwell et al., 2003). Mice with brain restricted deletion of PEX13 ("PEX13 brain mutants") have a milder phenotype and have been useful for investigating postnatal brain development; these mutants have impaired granule cell layer migration, abnormal cerebellar Purkinje cell differentiation (Muller et al., 2011) and abnormalities of the serotonergic system (Rahim et al., 2014). Despite the significant findings from these and other mouse models, the cellular mechanisms underlying ZS neuropathology are still unresolved (Crane, 2014).

Although primarily a peroxisome deficiency disorder, a potential role for mitochondria in ZS neuropathogenesis has been evident since early findings of mitochondrial abnormalities in ZS patients (Goldfischer et al., 1973). Mitochondria and peroxisomes carry out a number of similar, non-redundant functions, such as fatty acid $\beta$-oxidation and detoxification of reactive oxygen species (ROS), and, in response to metabolic cues, undergo rapid remodelling that 
involves common components of fission machinery (Schrader, 2006), which together suggest an important inter-dependency. Research on the above-mentioned PEX gene mouse models of ZS has also demonstrated significant changes to mitochondrial structure and function in various tissues, such as mitochondrial proliferation and dysmorphology, respiratory chain dysfunction, increased levels of (mitochondrial) SOD2, and increased levels of mitochondriagenerated reactive oxygen species (ROS) (Baumgart et al., 2001, Krysko et al., 2007, Muller et al., 2011).

In the context of the neuropathology of ZS, we hypothesize that mitochondrial dysfunction is a plausible consequence of peroxisome deficiency that may be mediated by an oxidative stress-dependent pathway of organelle remodelling. In this regard, there is growing evidence of an important metabolic link between the peroxisome and mitochondrial redox systems (Walton and Pizzitelli, 2012, Wang et al., 2013). The present study addresses mitochondrial changes arising from PEX13 deficiency, to investigate a possible association between oxidative stress, mitochondrial structure and function, and cellular indicators of tissue pathology. 


\section{Materials and methods}

\section{Animals}

PEX13 brain mutants, PEX13 knockout mice, and littermate wild-type mice, were generated and genotyped as reported previously (Maxwell et al., 2003, Muller et al., 2011). Mouse genetic modification was approved by the Griffith University Institutional Biosafety Committee (NLRD/21/07), and animal experimentation was approved by the Griffith University Animal Ethics Committee (BBS/02/09/AEC; ESK/03/13/AEC). The "aged" mouse used in these experiments was a 1 year-old wild-type C57BL/6J, the background strain of the PEX13 mutants.

\section{Perfusion-fixation of mouse brain}

For immunofluorescence analysis, brains were collected as follows: P20 animals were first anaesthetized with $80 \mathrm{mg} / \mathrm{kg}$ ketamine and $10 \mathrm{mg} / \mathrm{kg}$ xylazine, then perfused transcardially with $10 \mathrm{mM}$ phosphate-buffered saline, $\mathrm{pH} 7.4$ (PBS) containing $0.5 \%$ sodium nitrite at a rate of $0.5 \mathrm{~mL} / \mathrm{min}$ to removed blood, followed by perfusion with modified Zambonie's fixative reagent (2\% paraformaldehyde, $0.2 \%$ picric acid, $0.1 \mathrm{M}$ PBS, $\mathrm{pH} 7.2$ ). Brains were then removed and retained in fixative overnight in vacuo. The following day, fixed brains were rinsed with PBS three times for 30 min to remove fixative and stored in PBS/ $0.5 \%$ azide (PBS/azide) at $4^{\circ} \mathrm{C}$ for later processing.

\section{Tissue cryo-processing and immunofluorescence analysis}

Brains were washed twice with PBS for 30 min then placed in $30 \%$ sucrose containing PBS/azide overnight at $4^{\circ} \mathrm{C}$. Brains were then passed through a series of graded OCT solutions $(20 \%, 30 \%, 50 \%, 70 \%$ OCT, prepared in $30 \%$ sucrose in PBS/azide, and finally $100 \%$ OCT $)$ for 60 min each and then moulded in cryomolds $(25 \mathrm{~cm} \times 22 \mathrm{~cm})$ with $100 \%$ OCT solution and stored at $-80^{\circ} \mathrm{C}$ until sectioned. Whole brain was sectioned using a Leica $\mathrm{CM}$ 3050s cryostat and sections were collected in a 24-well plate containing PBS/azide as freefloating sections or on supra-frost slides as adherent sections, and kept at $4^{\circ} \mathrm{C}$ and $-80^{\circ} \mathrm{C}$ 
respectively, until further analysis. $40 \mu \mathrm{m}$ thick coronal brain sections from 3 wild-type and 3 PEX13 brain mutants (6 sections for each) were carefully matched through reference to the Paxinos and Franklin mouse brain atlas (Paxinos and Franklin, 2001). Brain sections were rinsed with PBS containing $0.1 \%$ Triton X-100 (PBS/Triton) and permeabilized using 100\% DMSO (Sigma) for 20min. To block non-specific binding sites on tissue, sections were incubated in blocking buffer (PBS/Triton X-100 containing 10\% donkey serum (v/v) for $1 \mathrm{~h}$ at room temperature. Multiple combinations of primary antibodies were used: mouse anti-GFAP, at a dilution of 1:800 (Sapphire Biosciences, Waterloo, NSW, Australia); rabbit anti-IBA-1, 1:2000 (Wako Chemicals, Richmond, VA, USA); goat anti-TPH2 (Everest Biotech Ltd, Oxfordshire, UK), rabbit anti-TPH2 (ThermoFisher Scientific, Inc, Rockford, IL, USA), rabbit anti-5HT (Sigma Aldrich Ltd, NSW, Australia); rabbit anti-SOD2, 1:500 (Abcam plc, Cambridge, UK), sheep anti-SOD2, 1:1000 (EMD Millipore, Billerica, MA, USA), rabbit antiNOS, 1:500 (Merck Millipore, Billerica, Massachusetts, USA), mouse anti-PMP70, 1:600 (Maxwell et al., 2003), rabbit anti-PEX14 (Maxwell et al., 2003), rabbit anti-PGC-1a (Santa Cruz Biotech Inc. Dallas, Texas USA). Antibodies were diluted in blocking buffer and incubated with sections overnight at $4^{\circ} \mathrm{C}$. Sections were then washed thoroughly in PBS/Triton and incubated with the relevant Alexa Fluor-conjugated secondary antibodies diluted in PBS/Triton, for $3 \mathrm{~h}$ at room temperature - donkey anti-rabbit 488 or 594 (1:400; Life Technologies, Carlsbad, CA, USA); donkey anti-goat 594 or 488 (1:400, Invitrogen); donkeyanti-goat 650, 1:50 (Abcam plc, Cambridge, UK), donkey-antisheep 594 (1:400; Life Technologies Carlsbad, CA, USA), donkey-anti-mouse 594 (1:400; Life Technologies, Carlsbad, CA, USA). To reduce non-specific background staining from secondary antibodies, multiple washing steps (up to 10 times depending on the antibody) using PBS/TritonX-100 were employed.

\section{Preparation and analysis of cultured mouse embryonic fibroblasts}

Mouse embryonic fibroblasts (MEFs) were prepared from stage 14 embryos and cultured as described previously (Nguyen et al., 2006), except that culture flasks were pre-coated with $2 \%$ gelatin to enhance cell adhesion. Immunofluorescence analysis of cultured MEFs was also as described (Nguyen et al., 2006). Other assays used fluorescence microscopy of 
MEFS following incubation with specific probes: mitochondrial morphology and distribution were assessed following incubation with $200 \mathrm{nM}$ MitoTracker Red CMXRos (Invitrogen) for 30 min at $37^{\circ} \mathrm{C}$, and rabbit SOD2 antibody (1:200) (Abcam plc, Cambridge, UK); levels of mitochondrial superoxide were assessed following incubation with $5 \mu \mathrm{M}$ MitoSOX (Invitrogen) for $15 \mathrm{~min}$ at $37^{\circ} \mathrm{C}$; mitochondrial membrane potential was measured following incubation with $1 \mu \mathrm{M} \mathrm{JC}-1$ dye (Invitrogen) for $30 \mathrm{~min}$ at $37^{\circ} \mathrm{C}$. As positive controls for superoxide generation and membrane potential, MEFs were incubated with $2 \mu \mathrm{M}$ rotenone for 30 min at $37^{\circ} \mathrm{C}$. Partial inhibition of catalase enzymatic activity was achieved by incubating wild-type MEFS with 2 mM 3-amino-1,2,4,-triazole (K\&K laboratories) for $4 \mathrm{~h}$ at $37^{\circ} \mathrm{C}$ (Walton and Pizzitelli, 2012). Catalase activity in MEF homogenates was determined using a spectrophotometric analysis as previously described (Crane et al., 1985). To assess the direct effect of an oxidant on mitochondria, $250 \mu \mathrm{M}$ hydrogen peroxide was added to culture medium of wild-type MEFs for $2 \mathrm{~h}$ (Stamer et al., 2002). Antioxidant effect was tested following addition of $1 \mathrm{mM} \mathrm{N}$ acetylcysteine to culture medium for $24 \mathrm{~h}$ (Kageyama et al., 2012).

\section{Fluorescence microscopy}

Images were captured with an Olympus FV1000 confocal laser scanning microscope.

\section{Morphological and distributional analyses of mitochondria}

Distributional analysis of mitochondria in brain sections was achieved by immunofluorescence analysis of SOD2 as a mitochondrial marker and Tph2 or Tuj-1 as cell markers. Z-stack images (0.1-0.2 $\mu \mathrm{m}$ optical planes, $1600 \times 1600$ resolution) were captured using constant exposure times, with 40-50 cells analysed per animal. A blind threshold was applied for each fluorescence channel and the Polygonal tool of image J (National Institutes of Health, Bethesda, MD, USA) was used to outline areas of neuronal cytoplasm and axons in midbrain. For each image, fluorescence channels were split and inverted. The red channel (Tph2 or Tuj-1 fluorescence) was used to outline somata and axons, which were then further used as templates for the second fluorescence green channel (e.g. SOD2 to identify mitochondria) and using the Analysed Particles feature of Image $\mathrm{J}$ to determine the relative area occupied by these organelles. 
Mitochondrial distribution was similarly analysed in cultured MEFs following incubation with MitoTracker Red CMXRos, as described above.

Imaris software (Bitplane, Inc. Zurich Switzerland) and Image J were used to quantify mitochondrial distribution and morphology. To analyse mitochondrial parameters, Zstack images of 0.1-0.2 $\mu \mathrm{m}$ thin optical sections were taken at high magnification of unlabelled or TPH2-labelled neurons. For morphological analysis, z-stacks were opened in Imaris to construct 3D images. Regions of interest, of defined diameter, were randomly selected from areas of cytoplasm abundant in mitochondria. For volume reconstruction, we used the IsoSurface volume rendering Surpass module to automatically capture mitochondrial structures in three dimensions and reconstruct images as 2D or 3D models. This feature also allowed measurement of mitochondrial volume per cell in both whole tissue sections and single cells that had been "digitally extracted" from sections. 2D volume reconstruction $(x-y)$ was also achieved by using the 3D viewer plugin (ImageJ). For comparative analyses, mitochondrial volume was normalized to cell volume.

In some applications, individual neuron cell area was calculated using image $\mathrm{J}$.

\section{Analysis of oxidative damage}

Lipid peroxidation and DNA oxidation were measured by immunofluorescence using antibodies to the lipid oxidation product 4-hydroxy-2-nonenal (HNE) and to the DNA oxidation product 8-hydroxyguanosine (8-OHG), respectively, on coronal sections of midbrain. For optimization of results, primary antibodies were tested with 3 different fluorescently tagged secondary antibodies to check for cross-reactivity and specificity, and unstained sections were used to check for auto-fluorescence. Experimental results were validated using a 1 yearold wild-type mouse as a positive control. To minimise background fluorescence, multiple washing steps were employed after incubation with primary and secondary antibodies. Fluorescence-positive cells were quantified using imageJ. 
Densitometric measurements

To measure comparative immunofluorescence intensities for SOD2, 8-OHG and HNE in different areas of the brain, images were captured by applying gain and offset adjustment options of the microscope to set the background fluorescence, on a region lacking detectable fluorescence, and to adjust/minimise the saturation independently for each fluorescence channel. After processing, images from all channels were merged. Images were taken at constant exposure time to allow comparison of matched specimens, and fluorescence intensity was quantified using imageJ following conversion to grey scale.

Western blot analysis

Brain tissue homogenates were prepared and analysed by SDS-PAGE and Western blot analysis as previously described (Muller et al., 2011). Antibodies were used at the following dilutions: PGC-1a (H-300; Santa Cruz Biotechnology Inc) 1/200; SOD2 (Abcam plc, Cambridge, UK) 1/3,000; $\beta$-tubulin, (Abcam, ab6046) 1/10,000; PEX13 (Bjorkman et al., 2002) $1 / 1,000$.

\section{Statistical analyses}

Statistical comparison between groups (wild-type vs brain mutant) was carried out using the Student's t-test. 


\section{Results}

Mitochondrial changes in neurons in brain of PEX13 brain mutants

The possibility of mitochondrial changes contributing to the neuropathology of PEX13deficient mice was primarily addressed using microscopic analysis of brain sections from P20 mice, to attempt to represent the in vivo situation. In some specific experiments we focused on the mid-brain as we recently demonstrated abnormality of serotonergic neurons in PEX13 brain mutants (Rahim et al., 2014). As a primary approach, we investigated changes to levels and distribution of superoxide dismutase-2 (SOD2, or Mn-SOD), which is exclusively located in mitochondria in mice (Karnati et al., 2013) and is responsible for removal of mitochondriagenerated superoxide radical in brain (Flynn and Melov, 2013). We used SOD2 immunofluorescence (IF) analysis to assess changes to the mitochondrial compartment and mitochondrial distribution and as a semi-quantitative measure of SOD2 protein levels per se.

All brain regions examined (olfactory bulb, brain stem, hippocampus, cerebral cortex, raphe nuclei) showed apparent 2-3-fold increases in SOD2 IF levels in PEX13 brain mutants when compared to wild-type mice (Fig. 1A). However, Western blot analysis indicated no statistically significant changes ( $p<0.05$; SOD2 normalised to $\beta$-tubulin) in the levels of SOD2 protein in extracts from a number of gross brain regions (Fig. 1B) or in total brain extracts (data not shown).

To explore this apparent inconsistency, a more detailed analysis of the mid-brain raphe region was undertaken. IF analysis again demonstrated an apparent 2.5 -fold increase in the volume of the SOD2-labelled mitochondrial compartment in neurons (Fig. 1C, D). We consequently investigated mitochondrial distribution in cells using Z-stack Imaris software analysis of SOD2 IF images. We demonstrated that, whereas mitochondria were dispersed throughout both cell somata and axons of wild-type mice, in PEX13 brain mutants they were largely restricted to somata (Fig. 1C, E). In addition, axon length for the mutants was reduced by about half (Fig. $1 F$ ), indicating a general loss of axonal integrity throughout the brain. A possible, at least 
partial, explanation of the apparent increase in SOD2 immunofluorescence may therefore be the redistribution of mitochondria to somata.

Using serotonergic cells (TPH2-positive) as a specific, identifiable class of neurons, we analysed mitochondrial morphology using Imaris analysis of SOD2-positive structures. Two dimensional analysis indicated the presence of larger mitochondrial structures in the PEX13 mutant cells (Fig. 2A). Using the Imaris software to measure the volume of individual (SOD2labelled) mitochondrial compartments confirmed a pronounced shift in the profile of brain mitochondria to much larger structures in the mutant mice (Fig. 2B). Based on this volume analysis, the total mitochondrial compartment of mutant cells was increased 5-fold (5.22 \pm 0.14-fold that of wild-type cells, $p<0.01$; mitochondrial volume normalised to cell volume). In this respect, it should be noted that the size of TPH2-positive cells in mutants was also increased $(1.25 \pm 0.13$-fold that of wild-type cells; $p<0.05)$, whereas we have previously reported that the total number of TPH2-positive cells is decreased 2-fold in PEX13 brain mutants (Rahim et al., 2014).

To confirm and expand the findings observed for brain sections, we undertook a parallel analysis of cultured fibroblasts from PEX13 knockout stage 14 mouse embryos, using a more comprehensive range of reagents available for cultured cells. Mitochondria were analysed using SOD2 IF and MitoTracker Red fluorescence labelling. MitoTracker Red analysis alone showed that, as for brain sections of PEX13 brain mutants, mitochondria in the majority of PEX13-deficient MEFs were distributed in a more peri-nuclear pattern than for wild-type MEFs (Fig 2C). To investigate potential changes to mitochondrial morphology, while minimising potential bias due to mitochondrial distribution, we selected those rare PEX13deficient MEFs whose cell morphologies were most similar to wild-type MEFs and specifically those with evident polarity and partial cytoplasmic distribution of mitochondria. The first significant finding from these experiments was the co-localization of MitoTracker Red fluorescence and SOD2 IF in cells (Fig. 2D), validating the use of SOD2 labelling as a measure of the mitochondrial compartment in these cells, and by inference in brain sections. SOD2 IF intensity in cell somata was 2.3-fold higher in PEX13-deficient MEFs than wild-type 
MEFs ( $p<0.05 ; n=100$ cells). The second significant finding was that $2 D$ analysis of the MitoTracker images demonstrated the presence of aberrant mitochondrial morphologies - a combination of larger, tangled and "donut"-shaped structures - in the PEX13-deficient MEFs (Fig. 2 D, E). The results from analysis of cultured PEX13-deficient MEFs therefore corroborate the findings from analysis of brain sections from PEX13 brain mutants in demonstrating abnormal mitochondrial distribution and morphology.

Mitochondrial changes in PEX13-deficient MEFs are consistent with a response to oxidative stress

Given the above results, we analyzed cultured MEFs from PEX13-deficient mice to determine possible changes to mitochondria that might reflect an oxidative challenge. We used MitoSOX to detect mitochondrial superoxide (Robinson et al., 2008) and JC-1 fluorescence as a measure of the mitochondrial membrane potential (Smiley et al., 1991). These probes were first validated using treatment of wild-type MEFs with rotenone, which inhibits complex 1 and depolarizes the mitochondrial membrane (Moon et al., 2005). Rotenone treatment increased the levels of MitoSox fluorescence and decreased the levels of JC-1 red aggregate fluorescence by more than 4 -fold ( $p<0.05, n=100$ cells) (Fig. 3A), consistent with increased superoxide levels and decreased mitochondrial membrane potential, respectively.

Application of these probes to PEX13-deficient and wild-type mice MEFs demonstrated that relative levels of MitoSOX fluorescence were 4.0-fold higher in PEX13-deficient MEFs $(p<0.05 ; n=100$ cells) (Fig. 3B), and the relative levels of JC-1 red fluorescence were 3.0-fold lower in the PEX13-deficient MEFs ( $p<0.05 ; n=100$ cells) (Fig. 3C). These results are consistent with increased generation of mitochondrial reactive oxygen species, and collapse of the mitochondrial membrane potential.

In a separate approach to determine if loss of peroxisomal redox metabolism caused by peroxisome deficiency would impact mitochondria, wild-type MEFs were incubated with 3amino-1,2,4-triazole (3-AT), a specific inhibitor of catalase. 3-AT treatment led to a $60 \%$ 
decrease in catalase activity. Under these conditions, there was a distinct shift in mitochondria to a more perinuclear distribution, a 3.8-fold increase in the relative levels of MitoSOX fluorescence, and a 4.9-fold decrease in the relative levels of JC-1 red fluorescence ( $p<0.05, n=100$ cells). 3-AT treatment also led to the accumulation of mitochondria with aberrant morphologies similar to those seen in PEX13-deficient MEFs (Fig. 3D). As an adjunct to these experiments, we assessed the effect of addition of $250 \mu \mathrm{M}$ hydrogen peroxide to the culture medium of wild-type MEFs. The results obtained were similar to those from 3-AT treatment in terms of an increase in MitoSOX fluorescence and aberrant mitochondrial morphologies (Fig. 3E). Finally, we treated PEX13-deficient MEFs with $1 \mathrm{mM} \mathrm{N}$ acetylcysteine to investigate the effect of antioxidant treatment. We demonstrated rescue of the phenotype - the majority of MEFs ( $>73 \% ; n=200$ cells) exhibited improved polarity, and mitochondria were distributed throughout cellular processes, showed normal levels of MitoSOX fluorescence, and displayed near-normal morphology (Fig 3F). Taken together, these results strongly suggest that oxidative stress is at least partially causative of the mitochondrial changes in PEX13-deficient cells, and implicate the loss of peroxisomal catalase and resultant accumulation of hydrogen peroxide in this process.

Given the observed mitochondrial changes and associated indications of oxidative stress, we

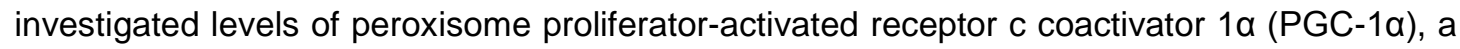
master regulator of mitochondrial biogenesis and expression of ROS-detoxifying enzymes, including SOD2 (Austin and St-Pierre, 2012). Western blot analysis of brain tissue extracts demonstrated a 1.7-fold increase in levels of PGC-1a for the PEX13 brain mutants (Fig. 3G, H), which suggests a compensatory response to oxidative stress and/or mitochondrial dysfunction.

\section{Oxidative damage in midbrain}

If, as suggested by the findings above, peroxisome deficiency leads to a sustained mitochondrial redox challenge, a predicted downstream effect would be oxidative damage to cells. In testing this, we again focused on the midbrain. We used immunofluorescence (IF) 
analysis of DNA oxidation and lipid peroxidation using antibodies to 8-hydroxyguanosine (8OHG), a product of DNA (and RNA) oxidation (Nunomura et al., 1999), and 4-hydroxynonenal (HNE) (Uchida and Stadtman, 1992), a product of lipid peroxidation, respectively. For validation of these analyses we tested in parallel a 1 year-old wild-type mouse to provide a positive control, as aging in mice has been shown to be accompanied by oxidative damage in brain (Hamilton and Holscher, 2012).

Levels of both 8-OHG and HNE IF were low in wild-type mice, and comparatively much higher for the PEX13 brain mutants (Fig. 4A). Quantification demonstrated a more than 5 -fold increase in the number of cells that were counted as positive for 8-OHG IF (Fig. 4B) and HNE IF (Fig. 4C). 8-OHG IF was also higher in serotonergic cells (TPH2-positive) of mutants (Fig. 4D). IF detected using the 8-OHG antibody was evident as prominent staining of cell nuclei (representing DNA damage) and minor diffuse cytoplasmic staining, the latter consistent with the manufacturer's reported detection of oxidised RNA, and possibly oxidised mitochondrial DNA, by this antibody. HNE IF was predominantly cytoplasmic. Both the 8-OHG IF (Fig. 4E) and HNE IF (Fig. 4F) localised predominantly to those cells with high levels of SOD2, suggesting concomitant oxidative damage to nucleic acids and lipids in these cells, and a correlation to mitochondrial changes.

Compared to the low levels of 8-OHG and HNE IF for the wild-type mouse brains, the aged mouse control showed high levels of 8-OHG and HNE IF (Fig. 4A), indirectly validating the IF analyses above; the 8-OHG fold-changes were similar to those previously reported using immunohistochemistry (Hamilton and Holscher, 2012).

These results, showing increased oxidative damage in the PEX13 brain mutants, are consistent with a mitochondrial response to a cellular redox challenge. 
Glial cell changes

Recent studies have revealed that increased oxidative stress triggers activation of astrocytes (Noh et al., 2013). Given our recent findings demonstrating a strong glial response in brains of PEX13 brain mutants (Muller et al., 2011, Rahim et al., 2014), and the evidence here of oxidative damage, we investigated possible mitochondrial changes and oxidative damage in astroglia.

PEX13 brain mutants exhibited pronounced reactive astrogliosis (Fig. 5A), as demonstrated by a more than 4-fold increase in IF levels of the marker GFAP over wild-type controls (Fig. 5B), confirming our previous report (Rahim et al., 2014). SOD2 IF in astroglia was increased in parallel with increased GFAP IF (Fig. 5A). Oxidative damage (8-OHG and HNE IF) was also increased in astroglia (Fig. $5 \mathrm{C}-\mathrm{F}$ ). These results suggest that the changes described for neurons in the PEX13 brain mutant are also true of glial cells. 


\section{Discussion}

The research described here was based on two potentially related premises. The first premise was that mitochondrial changes are associated with the neuropathology of ZS. This was inferred from previous research that identified mitochondrial functional and structural changes in ZS patients (Goldfischer et al., 1983, Hughes et al., 1990) and mouse models of ZS (Baumgart et al., 2001, Muller et al., 2011). The second premise was that oxidative stress triggers the mitochondrial changes. Given the evidence indicating that mitochondria are targets of peroxisome-derived oxidants (Walton and Pizzitelli, 2012, Wang et al., 2013), we speculated that peroxisome deficiency may alter cellular redox balance in a way that adversely impacts mitochondrial biogenesis, dynamics and function.

Our main findings from analysis of brains of PEX13 brain mutants demonstrated a significantly expanded mitochondrial compartment and structural changes that imply abnormal mitochondrial dynamics, and predominately a pro-fusion state. The parallel studies on PEX13-deficient MEFs complemented these findings in demonstrating aberrant mitochondrial morphologies as well as increased mitochondrial superoxide levels and mitochondrial depolarisation, from which we infer that similar changes are also true of brains of the PEX13 mutants. We presume that the slightly different mitochondrial morphologies in PEX13-deficient brain and MEFs reflect the normal morphologies in these tissues - more rounded in brain, filamentous in fibroblasts (Youle and van der Bliek, 2012).

These findings therefore support mechanisms of neuropathogenesis that are associated with both mitochondrial defects and redox changes, in keeping with our hypothesis. Consistent with the hypothesis, the expanded mitochondrial compartment showed a shift to larger structures resembling the "donut" and "blob" forms recently described for cells undergoing oxidative stress, and identified as the predominant source of mitochondrial ROS (Ahmad et al., 2013). The primary source of the ROS predicted to trigger the changes proposed above was not identified in these experiments, but the loss of peroxisomal function is plausible, and is supported by our findings that both 3-aminotriazole inhibition of catalase and hydrogen 
peroxide treatment resulted in a mitochondrial phenotype similar to that seen in PEX13deficient cells. Of interest, the effect of hydrogen peroxide treatment was similar to that observed for fibroblasts from mice lacking the mitochondrial fission factor Drp1, i.e. conversion from elongated tubules to large spheres (Kageyama et al., 2012). Consistent with oxidative stress being a primary driver of these changes, we found that treatment of PEX13deficient MEFS with the antioxidant $\mathrm{N}$-acetylcysteine rescued the mitochondrial phenotype. We therefore suggest a model in which mitochondria have responded to an oxidative stress challenge - a result of peroxisome deficiency - which has led to increased mitochondrial ROS generation and the formation of aberrant mitochondrial morphologies. However, recent research has also demonstrated that PEX13 deficiency leads to defective mitophagy (M. Y. Lee, unpublished observations), thus providing an alternative explanation for the accumulation of larger mitochondrial structures. Potentially, the explanation for the different mitochondrial morphologies involves a combination of these cellular stresses.

A summary of the findings is therefore proposed to include the following events. PEX13 deficiency leads to peroxisome-derived mitochondrial oxidative stress, as well as defective mitophagy. This "double-hit" event leads to the retention of large, dysfunctional mitochondria that accumulate oxidative damage mediated by elevated levels of superoxide radical. This pro-fusion state of mitochondrial dynamics inhibits mitochondrial transport (Sheng and Cai, 2012), leading to depletion of mitochondria in cell processes and their concentration in somata. The upregulation of PGC1 1 , a master regulator of biogenesis of both mitochondria and peroxisomes (Austin and St-Pierre, 2012), represents a futile cellular response to generate functional organelles.

The state of chronic oxidative stress for the PEX13 brain mutants that is implied by the above findings would presage significant oxidative damage to the brain. Consistent with this scenario, we demonstrated high tissue levels of nucleic acid (8-OHG) and lipid (HNE) oxidation products. We have previously shown that the PEX13 brain mutants exhibit high levels of cell apoptosis in the midbrain and other brain regions (Rahim et al., 2014), and that cultured cerebellar neurons from PEX13-null mice exhibit increased rates of apoptosis (Muller et al., 2011). We speculate that these parameters are linked, i.e. cellular oxidative stress and 
defective mitochondrial dynamics are associated with enhanced cell degeneration; however the mechanisms involved remain to be elucidated. In highly polarized cells such as neurons, fission is required for the generation and distribution of mitochondria along cytoskeletal networks, as well as to allow formation of neuronal processes (reviewed in (Nunnari and Suomalainen, 2012). Our data, in demonstrating a loss of mitochondrial distribution along neuronal processes, infer a significant loss of mitochondrial function in neuronal processes, which may correlate with the observed loss of neuronal polarity in PEX13-deficient brain (Rahim et al., 2014).

Despite our data being more consistent with an oxidative stress model of mitochondrial change, it is not possible at this stage to discount a response to nutrient starvation (Nunnari and Suomalainen, 2012) resulting, for example, from loss of peroxisomal lipid metabolism. Mitochondrial biogenesis and remodeling may be due to nutrient starvation that causes increased mitochondrial respiratory flux - for example, a shift to more inter-connected, tubular mitochondrial forms as a result of unopposed mitochondrial fusion has been observed following nutrient depletion, and has been proposed as a protective mechanism to prevent mitophagy (Gomes et al., 2011, Rambold et al., 2011).

Although the work presented here has focused on changes to neurons, we also investigated effects on astroglia, given previous findings demonstrating glial cell changes in brains of PEX5- and PEX13-deficient mice (Hulshagen et al., 2008, Muller et al., 2011). In terms of mitochondrial remodeling and oxidative damage, we observed similar changes for astroglia to those seen for neurons. These findings raise the important, but vexed, question of the relative contribution and timing of neuronal and glial changes to the neuropathology of the PEX13 brain mutants.

In conclusion, we have established a finding of significant changes to the mitochondrial compartment in neuronal and glial cells of brains of PEX13 brain mutants. These changes, which include defective mitochondrial dynamics and distribution, are suggested to arise as a 
result of a redox imbalance caused by peroxisome deficiency, and which subsequently lead to mitochondrial-mediated changes that impact cell development and/or degeneration. 


\section{Figure Legends}

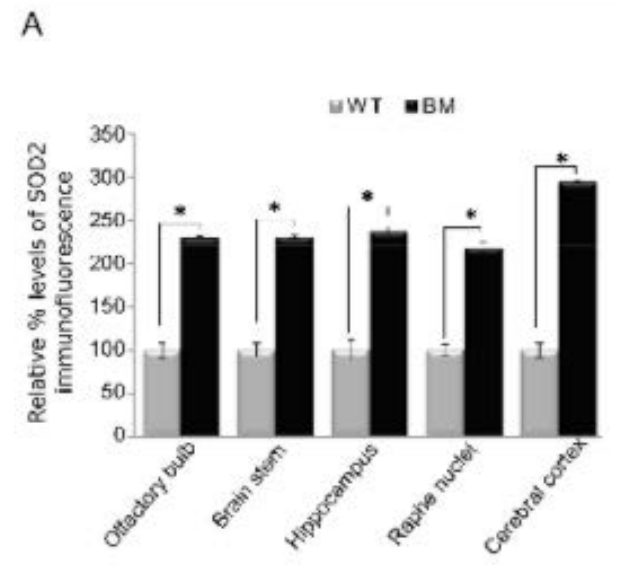

$\mathrm{C}$
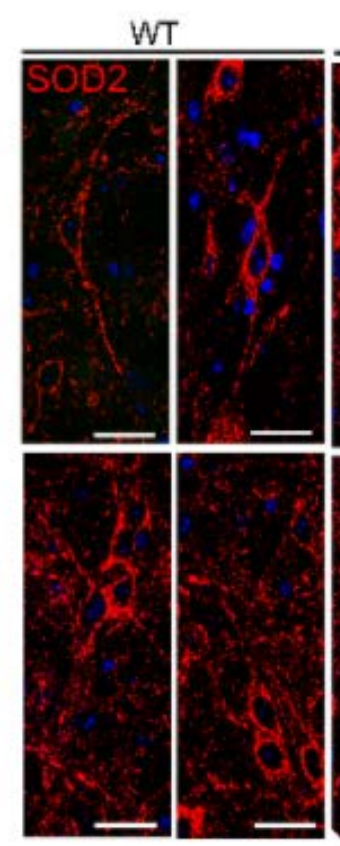

B
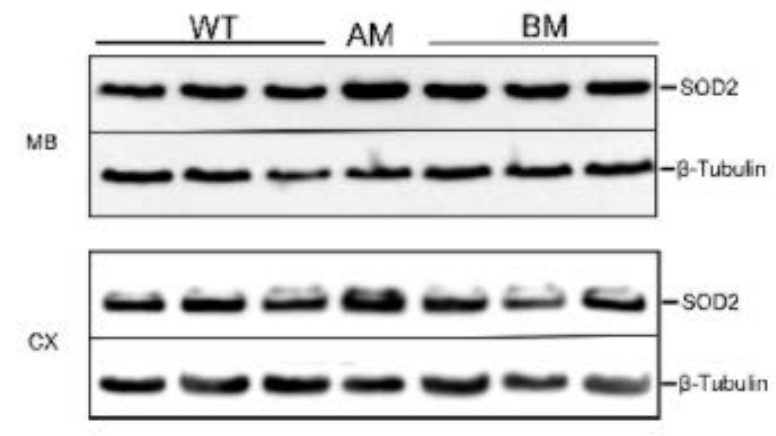

CB

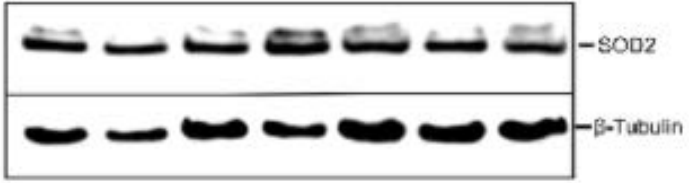

D

$E$
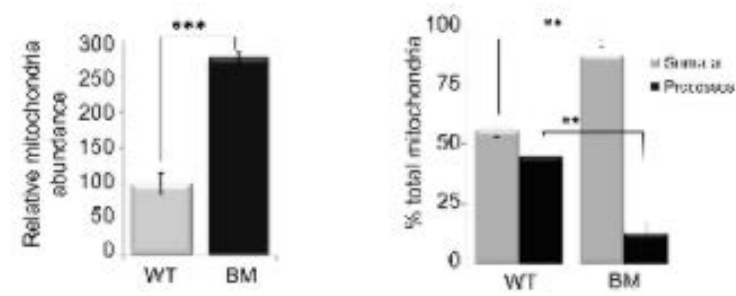

$\mathrm{F}$

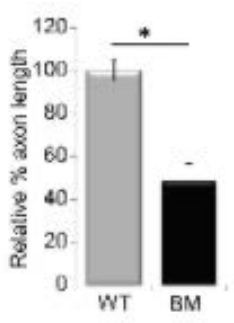

Fig. 1. SOD2 and mitochondrial changes in brain of PEX13 brain mutants. (A) Relative levels of SOD2 immunofluorescence captured at low magnification (10-20X) from matched sagittal sections of selected brain regions of P20 mice. Values are presented as a $\%$ of levels for wild-type mice. (B) Western blot analysis of SOD2 and $\beta$-tubulin in extracts of brain regions from wild-type and PEX13 brain mutant mice. A 1-year old wild-type mouse (AM) was used as a control. $25 \mu \mathrm{g}$ of protein was loaded for each sample. MB, "midbrain" region, but also including the hippocampus, thalamus, hypothalamus and pons; CX, cerebral cortex; CB, cerebellum. Panels C-F represent results from analysis of midbrain. (C) Levels and 
distribution of (SOD2-positive) mitochondria throughout neuronal somata and processes. Four examples are shown for both wild-type and PEX13 brain mutants. Cell nuclei were stained with DAPI (blue). Scale bars, 25 $\mu$ m. (D) Relative abundance of mitochondria (SOD2-positive structures), normalised to cell area, in neurons. (E) Quantitative analysis of mitochondrial distribution between somata and axons in neurons. Neuronal axons were selected manually for analysis using Imaris software. (F) Relative neuronal axon length. WT, wild-type; BM, PEX13 brain mutant. Data represent mean \pm SEM ( $n=3$ animals for each group). ${ }^{*} p<0.05$, ${ }^{* \star} p<0.01,{ }^{* \star *} p<0.001$. 


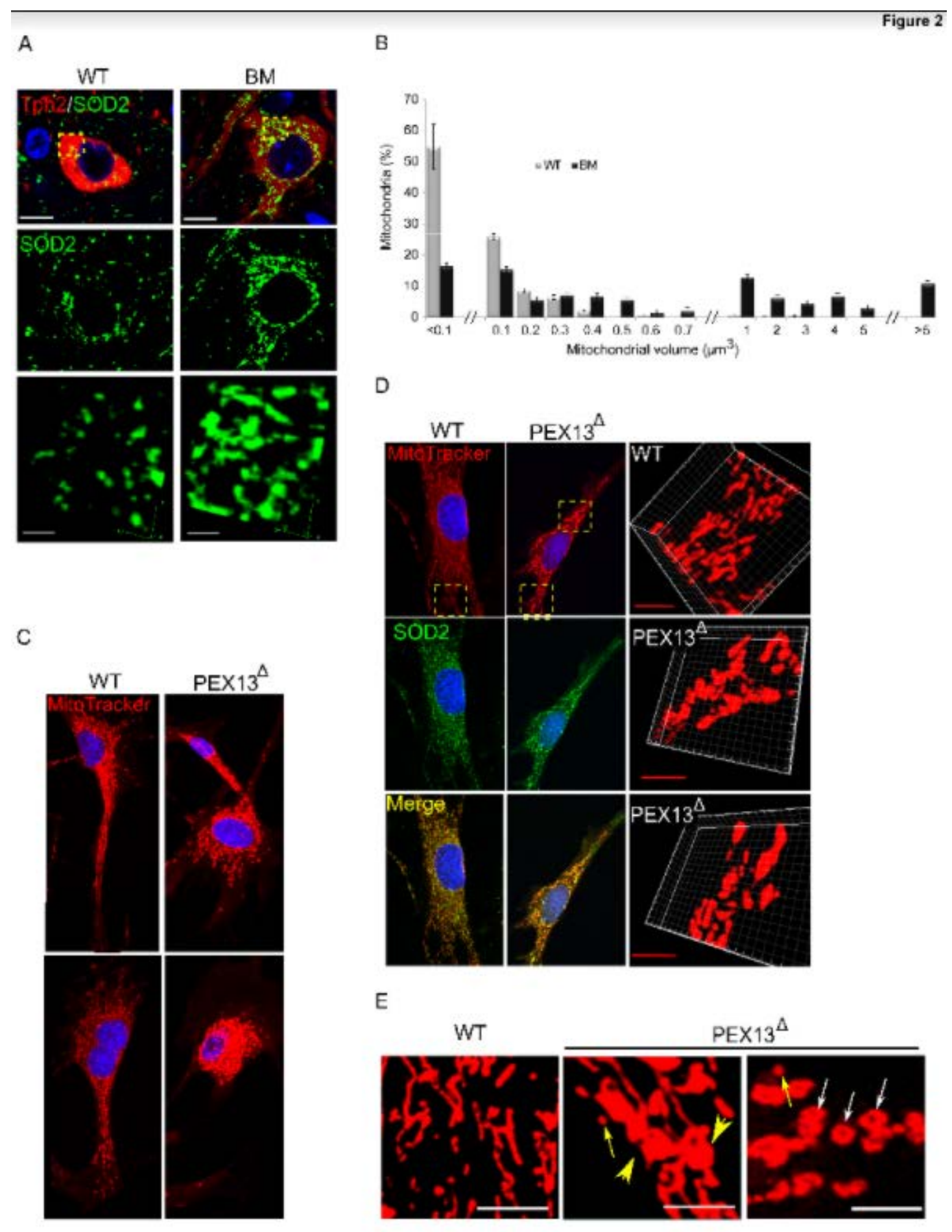

Fig. 2. Abnormal mitochondrial morphology in PEX13-deficient cells. (A) Representative image of SOD2 immunofluorescence (IF) in TPH2-positive serotonergic neurons in sagittal sections of P20 mice. Cell nuclei were stained with DAPI (blue). Scale bars, $10 \mu \mathrm{m}$. The bottom panels show mitochondrial morphology of cells from the top panels in 2D using Imaris software. Scale bars, $2.5 \mu \mathrm{m}$. (B) Profile of mitochondria in serotonergic neurons according to volume measured using Imaris software analysis of SOD2 IF. (C) MitoTracker red 
fluorescence analysis of cultured MEFs. Two images are presented for both WT and PEX13 ${ }^{\Delta}$ MEFS. (D) Co-localization of MitoTracker Red fluorescence and SOD2 IF in MEFs (left and middle panels). Right panels, 2D high resolution analysis of MitoTracker images (selected areas indicated in top left panels) to demonstrate mitochondrial morphology. Scale bars, $5 \mu \mathrm{m}$ (E) Higher resolution of representative mitochondrial morphologies in MEFs. WT MEFs display mainly tubular/reticular-shaped mitochondria and a low number of small spherical mitochondria. PEX $13^{\Delta}$ MEFs have a few small spherical structures as well as a range of aberrant morphologies: yellow arrows, small spherical mitochondria; yellow arrowheads, larger tangled/fused mitochondria; white arrows, donut-shaped mitochondria. Scale bars, 5 $\mu \mathrm{m}$. WT, wild-type, PEX13^ ${ }^{\wedge}$ PEX13-deficient. 


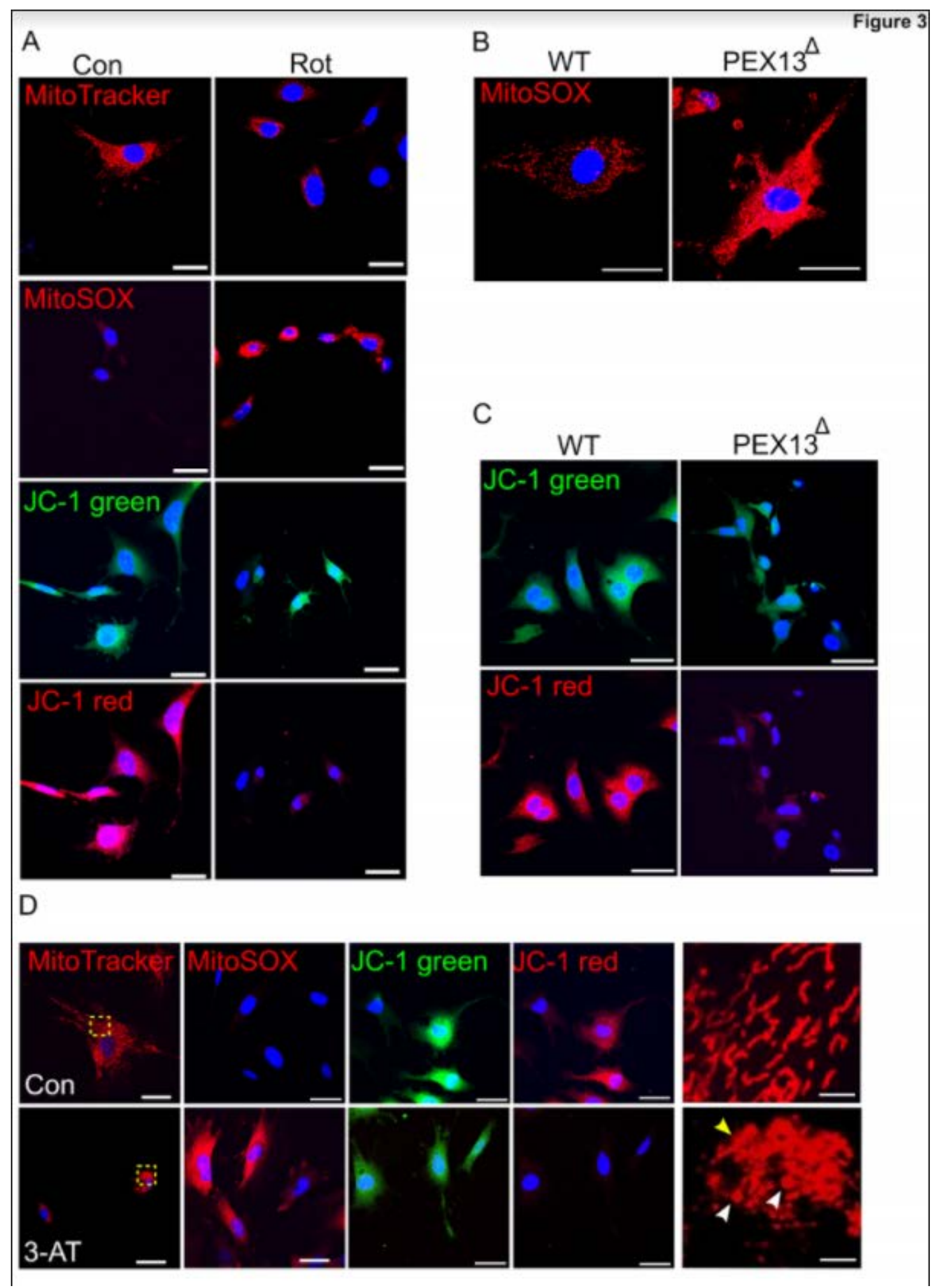

Fig. 3. Mitochondrial changes in cultured PEX13-deficient mouse embryonic fibroblasts (A) Wild-type MEFs were mock-treated (Con) or incubated with $2 \mu \mathrm{M}$ rotenone (Rot) to validate the MitoSox probe for measurement of mitochondrial superoxide and the JC-1 probe for measurement of mitochondrial membrane potential. JC-1 green fluorescence represents cellular uptake; JC-1 red fluorescence represents mitochondrial uptake and fluorescence 
shift, both dependent on mitochondrial membrane potential. Note: rotenone treatment resulted in cell shrinkage. (B) Relative levels of MitoSOX fluorescence in wild-type (WT) and PEX13-deficient (PEX13 ${ }^{\Delta}$ ) MEFs. (C) JC-1 green and red fluorescence in WT and PEX13 ${ }^{\Delta}$ MEFs. (D) Mitochondrial changes in wild-type MEFs pre-treated with 2 mM 3-amino-1,2,4,triazole (3-AT) for 4 h to inhibit catalase activity. Right panels, higher resolution images of mitochondria from indicated boxed areas. White arrowhead, donut-shaped mitochondria; yellow arrowhead, fused/tangled mitochondria. (E) Mitochondrial changes in wild-type MEFs following addition of $250 \mu \mathrm{M} \mathrm{H} \mathrm{O}_{2}$ to culture medium for $2 \mathrm{~h}$. Right panel, higher resolution images of mitochondria from indicated boxed area. White arrowheads, donut-shaped mitochondria; yellow arrowheads, fused/tangled mitochondria; yellow arrows, small spherical mitochondria. (F) Treatment of PEX13-deficient MEFs with $1 \mathrm{mM} \mathrm{N}$-acetylcysteine (NAC) for $24 \mathrm{~h}$ rescues the mitochondrial phenotype. Right panels, higher resolution images of mitochondria from indicated boxed areas. Scale bars for panels A-F main images, $50 \mu \mathrm{m}$; panels $D, E$ and $F$ magnified images, $5 \mu \mathrm{m}$. (G) Western blot analysis of PGC-1 $1 \alpha, \beta$-tubulin and PEX13 in brain tissue extracts from wild-type and PEX13 brain mutant mice. $25 \mu \mathrm{g}$ of protein was loaded for each sample. Predicted protein molecular masses shown at right were determined through comparison to Bio-Rad pre-stained molecular mass markers. $(\mathrm{H})$ Quantitative densitometric analysis of PGC-1 $\alpha$ band intensity from panel E normalised to $\beta$ tubulin. Values represent mean $\pm \mathrm{SEM},{ }^{*} \mathrm{p}<0.05$. WT, wild-type; BM, PEX13 brain mutant. 


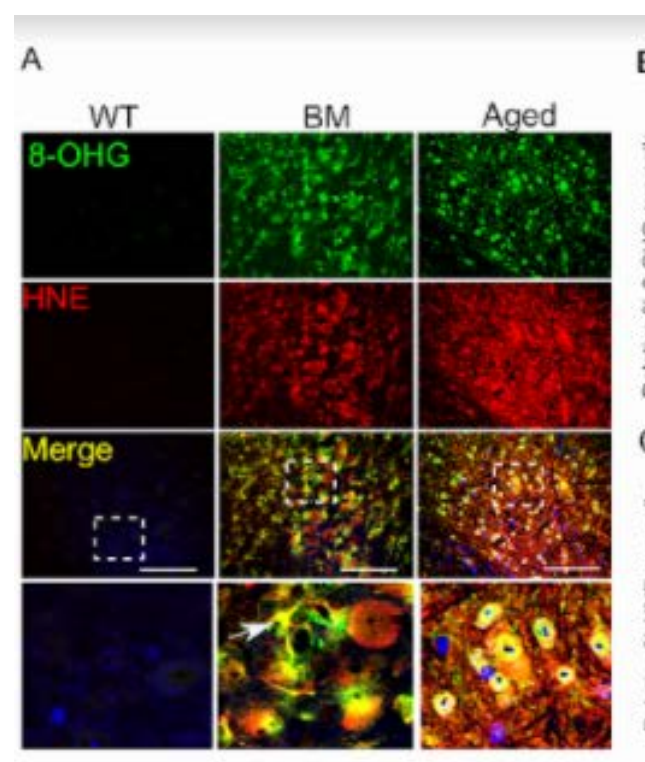

B D
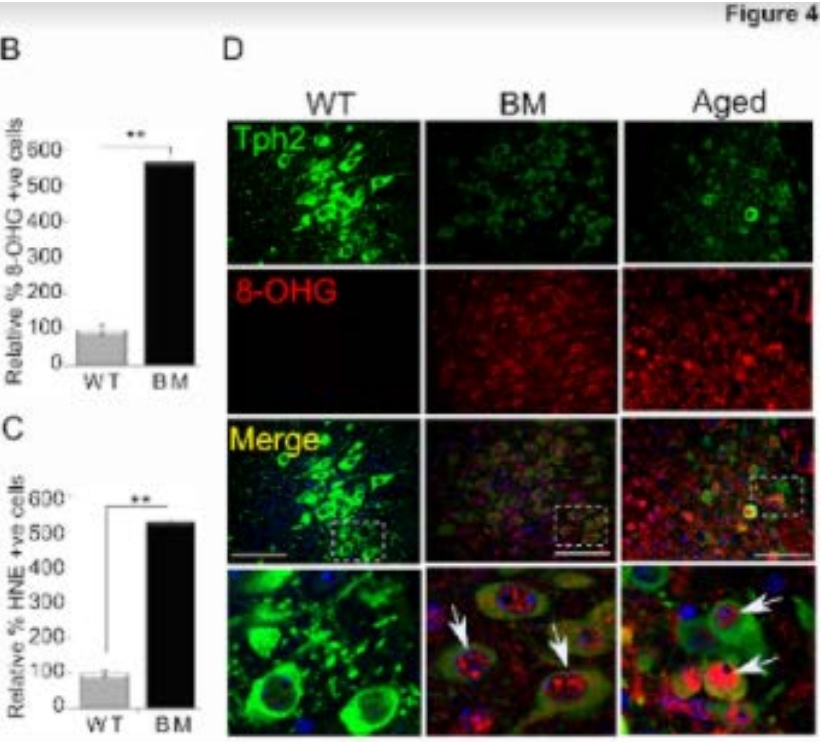

$E$

$\mathrm{F}$
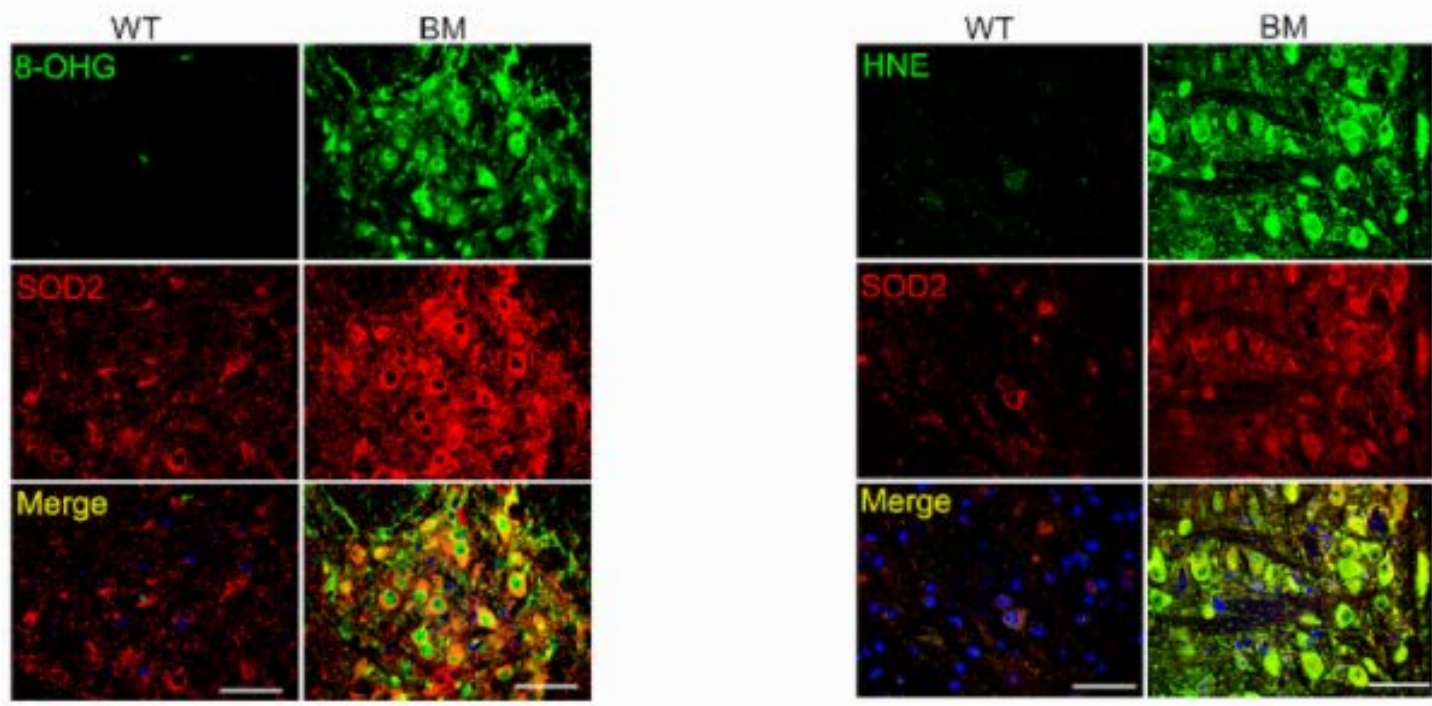

Fig. 4. Oxidative damage in PEX13 brain mutants. Oxidative damage in midbrain sections was assessed by immunofluorescence (IF) using antibodies to 8-OHG and HNE to detect DNA and lipid oxidation products, respectively. A 1 year-old wild-type mouse ("Aged") was used as a positive control. Nuclei were stained using DAPI (blue). (A) 8-OHG (green) and HNE (red) IF. Bottom panels, magnification of boxed areas depicted in the "Merge" images. The arrow depicts a cell with co-localised 8-OHG and HNE IF. (B,C) Quantification of 8-OHGpositive cells and HNE-positive cells, respectively, presented as a relative $\%$ of numbers in wild-type mice. (D) 8-OHG IF in TPH2-positive serotonergic neurons. Bottom panels, magnification of boxed areas depicted in the "Merge" images. The arrowheads indicate nuclear 8-OHG IF. (E) Co-localization of 8-OHG IF (green) with cells exhibiting high SOD2 IF 
(red) in midbrain. (F) Co-localization of HNE IF (green) with cells exhibiting high SOD2 IF

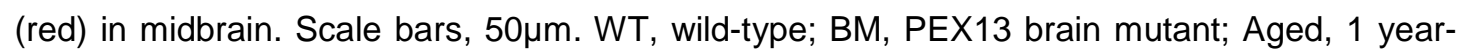
old wild-type mouse. Data represent mean \pm SEM for 3 animals for both WT and BM PEX13 brain mutant mice. ${ }^{* *} \mathrm{p}<0.01$.

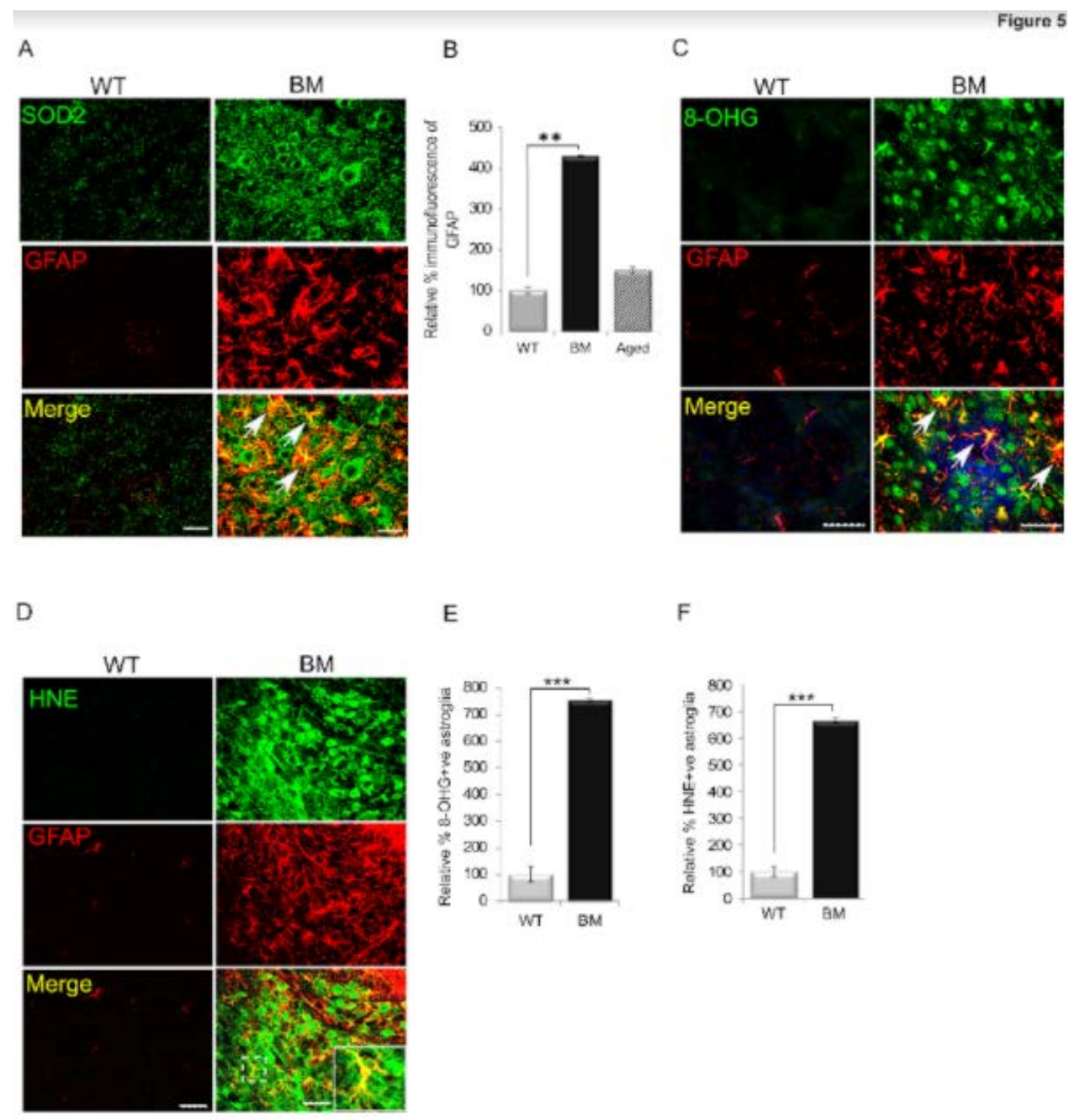

Fig. 5. Astroglial changes in brains of PEX13 brain mutants. (A) SOD2 and GFAP immunofluorescence (IF) in midbrain. Arrows indicate astroglia (GFAP-positive cells) with high SOD2 IF. (B) Quantification of GFAP IF. (C) 8-OHG IF (green) and GFAP IF (red) in cells of midbrain. Arrowheads indicate astroglia (GFAP-positive cells) with high 8-OHG IF. (D) HNE IF (green) and GFAP IF (red) in cells of the midbrain. Bottom "Merge" panel shows magnification of a GFAP-positive cell with high HNE IF. (E,F) Relative levels of 8-OHG- 
positive and HNE-positive astroglia (GFAP-positive) cells, respectively. Scale bars, 100 $\mu \mathrm{m}$. WT, wild-type; BM, PEX13 brain mutants; Aged, 1 year-old wild-type mouse control. Data represent mean \pm SEM for 3 animals for both WT and BM. ${ }^{* \star *} p<0.001$.

\section{ACKNOWLEDGEMENT}

We thank Alaina Hyunjung Jhin for carrying out the PGC-1a Western blot experiments and Ahsan-ur-Rehman for assistance with image analysis software.

\section{AUTHOR CONTRIBUTIONS}

R.S.R., M.C., C.C.N, A.C.B.M. and D.I.C. conceived and designed the experiments. R.S.R., M.C. and C.C.N. performed the experiments. R.S.R., MC, A.C.B.M. and D.I.C. wrote the paper.

\section{FUNDING}

This research was funded by Griffith University. 


\section{REFERENCES}

Ahmad T, Aggarwal K, Pattnaik B, Mukherjee S, Sethi T, Tiwari BK, Kumar M, Micheal A, Mabalirajan U, Ghosh B, Sinha Roy S, Agrawal A (2013) Computational classification of mitochondrial shapes reflects stress and redox state. Cell death \& disease 4:e461.

Austin S, St-Pierre J (2012) PGC1alpha and mitochondrial metabolism--emerging concepts and relevance in ageing and neurodegenerative disorders. Journal of cell science 125:4963-4971.

Baes M, Gressens P, Baumgart E, Carmeliet P, Casteels M, Fransen M, Evrard P, Fahimi D, Declercq PE, Collen D, van Veldhoven PP, Mannaerts GP (1997) A mouse model for Zellweger syndrome. Nat Genet 17:49-57.

Baumgart E, Vanhorebeek I, Grabenbauer M, Borgers M, Declercq PE, Fahimi HD, Baes M (2001) Mitochondrial alterations caused by defective peroxisomal biogenesis in a mouse model for Zellweger syndrome (PEX5 knockout mouse). Am J Pathol 159:14771494.

Bjorkman J, Gould SJ, Crane DI (2002) Pex13, the mouse ortholog of the human peroxisome biogenesis disorder PEX13 gene: gene structure, tissue expression, and localization of the protein to peroxisomes. Genomics 79:162-168.

Bottelbergs A, Verheijden S, Hulshagen L, Gutmann DH, Goebbels S, Nave KA, Kassmann C, Baes M (2010) Axonal integrity in the absence of functional peroxisomes from projection neurons and astrocytes. Glia 58:1532-1543.

Bottelbergs A, Verheijden S, Van Veldhoven PP, Just W, Devos R, Baes M (2012) Peroxisome deficiency but not the defect in ether lipid synthesis causes activation of the innate immune system and axonal loss in the central nervous system. Journal of neuroinflammation 9:61.

Crane DI (2014) Revisiting the neuropathogenesis of Zellweger syndrome. Neurochemistry international 69:1-8.

Crane DI, Hemsley AC, Masters CJ (1985) Purification of peroxisomes from livers of normal and clofibrate-treated mice. Analytical biochemistry 148:436-445.

Distel B, Erdmann R, Gould SJ, Blobel G, Crane DI, Cregg JM, Dodt G, Fujiki Y, Goodman JM, Just WW, Kiel JA, Kunau WH, Lazarow PB, Mannaerts GP, Moser HW, Osumi T, Rachubinski RA, Roscher A, Subramani S, Tabak HF, Tsukamoto T, Valle D, van der Klei I, van Veldhoven PP, Veenhuis M (1996) A unified nomenclature for peroxisome biogenesis factors. The Journal of cell biology 135:1-3.

Elgersma $Y$, Kwast L, Klein A, Voorn-Brouwer T, van den Berg M, Metzig B, America T, Tabak HF, Distel B (1996) The SH3 domain of the Saccharomyces cerevisiae peroxisomal membrane protein Pex13p functions as a docking site for Pex5p, a mobile receptor for the import PTS1-containing proteins. The Journal of cell biology 135:97109.

Erdmann R, Blobel G (1996) Identification of Pex13p a peroxisomal membrane receptor for the PTS1 recognition factor. The Journal of cell biology 135:111-121.

Faust PL (2003) Abnormal cerebellar histogenesis in PEX2 Zellweger mice reflects multiple neuronal defects induced by peroxisome deficiency. The Journal of comparative neurology 461:394-413.

Faust PL, Hatten ME (1997) Targeted deletion of the PEX2 peroxisome assembly gene in mice provides a model for Zellweger syndrome, a human neuronal migration disorder. The Journal of cell biology 139:1293-1305.

Flynn JM, Melov S (2013) SOD2 in mitochondrial dysfunction and neurodegeneration. Free radical biology \& medicine 62:4-12.

Goldfischer S, Moore CL, Johnson AB, Spiro AJ, Valsamis MP, Wisniewski HK, Ritch RH, Norton WT, Rapin I, Gartner LM (1973) Peroxisomal and mitochondrial defects in the cerebro-hepato-renal syndrome. Science 182:62-64.

Goldfischer S, Powers JM, Johnson AB, Axe S, Brown FR, Moser HW (1983) Striated adrenocortical cells in cerebro-hepato-renal (Zellweger) syndrome. Virchows Archiv A, Pathological anatomy and histopathology 401:355-361.

Gomes LC, Di Benedetto G, Scorrano L (2011) During autophagy mitochondria elongate, are spared from degradation and sustain cell viability. Nature cell biology 13:589-598. 
Gould SJ, Kalish JE, Morrell JC, Bjorkman J, Urquhart AJ, Crane DI (1996) Pex13p is an SH3 protein of the peroxisome membrane and a docking factor for the predominantly cytoplasmic PTs1 receptor. The Journal of cell biology 135:85-95.

Gould SJ, Valle D (2000) Peroxisome biogenesis disorders: genetics and cell biology. Trends Genet 16:340-345.

Hamilton A, Holscher C (2012) The effect of ageing on neurogenesis and oxidative stress in the APP(swe)/PS1(deltaE9) mouse model of Alzheimer's disease. Brain research 1449:83-93.

Hughes JL, Poulos A, Robertson E, Chow CW, Sheffield LJ, Christodoulou J, Carter RF (1990) Pathology of hepatic peroxisomes and mitochondria in patients with peroxisomal disorders. Virchows Archiv A, Pathological anatomy and histopathology 416:255-264.

Hulshagen L, Krysko O, Bottelbergs A, Huyghe S, Klein R, Van Veldhoven PP, De Deyn PP, D'Hooge R, Hartmann D, Baes M (2008) Absence of functional peroxisomes from mouse CNS causes dysmyelination and axon degeneration. The Journal of neuroscience : the official journal of the Society for Neuroscience 28:4015-4027.

Janssen A, Baes M, Gressens P, Mannaerts GP, Declercq P, Van Veldhoven PP (2000) Docosahexaenoic acid deficit is not a major pathogenic factor in peroxisome-deficient mice. Laboratory investigation; a journal of technical methods and pathology 80:31-35.

Kageyama Y, Zhang Z, Roda R, Fukaya M, Wakabayashi J, Wakabayashi N, Kensler TW, Reddy PH, lijima M, Sesaki H (2012) Mitochondrial division ensures the survival of postmitotic neurons by suppressing oxidative damage. The Journal of cell biology 197:535-551.

Karnati S, Luers G, Pfreimer S, Baumgart-Vogt E (2013) Mammalian SOD2 is exclusively located in mitochondria and not present in peroxisomes. Histochemistry and cell biology 140:105-117.

Kassmann CM, Lappe-Siefke C, Baes M, Brugger B, Mildner A, Werner HB, Natt O, Michaelis T, Prinz M, Frahm J, Nave KA (2007) Axonal loss and neuroinflammation caused by peroxisome-deficient oligodendrocytes. Nat Genet 39:969-976.

Krysko O, Hulshagen L, Janssen A, Schutz G, Klein R, De Bruycker M, Espeel M, Gressens $P$, Baes M (2007) Neocortical and cerebellar developmental abnormalities in conditions of selective elimination of peroxisomes from brain or from liver. J Neurosci Res 85:5872.

Liu Y, Bjorkman J, Urquhart A, Wanders RJ, Crane DI, Gould SJ (1999) PEX13 is mutated in complementation group 13 of the peroxisome-biogenesis disorders. American journal of human genetics 65:621-634.

Maxwell M, Bjorkman J, Nguyen T, Sharp P, Finnie J, Paterson C, Tonks I, Paton BC, Kay GF, Crane DI (2003) Pex13 inactivation in the mouse disrupts peroxisome biogenesis and leads to a Zellweger syndrome phenotype. Mol Cell Biol 23:5947-5957.

Moon Y, Lee KH, Park JH, Geum D, Kim K (2005) Mitochondrial membrane depolarization and the selective death of dopaminergic neurons by rotenone: protective effect of coenzyme Q10. Journal of neurochemistry 93:1199-1208.

Muller CC, Nguyen TH, Ahlemeyer B, Meshram M, Santrampurwala N, Cao S, Sharp P, Fietz PB, Baumgart-Vogt E, Crane DI (2011) PEX13 deficiency in mouse brain as a model of Zellweger syndrome: abnormal cerebellum formation, reactive gliosis and oxidative stress. Disease models \& mechanisms 4:104-119.

Nguyen T, Bjorkman J, Paton BC, Crane DI (2006) Failure of microtubule-mediated peroxisome division and trafficking in disorders with reduced peroxisome abundance. Journal of cell science 119:636-645.

Noh YH, Kim KY, Shim MS, Choi SH, Choi S, Ellisman MH, Weinreb RN, Perkins GA, Ju WK (2013) Inhibition of oxidative stress by coenzyme Q10 increases mitochondrial mass and improves bioenergetic function in optic nerve head astrocytes. Cell death \& disease 4:e820.

Nunnari J, Suomalainen A (2012) Mitochondria: in sickness and in health. Cell 148:11451159.

Nunomura A, Perry G, Pappolla MA, Wade R, Hirai K, Chiba S, Smith MA (1999) RNA oxidation is a prominent feature of vulnerable neurons in Alzheimer's disease. The Journal of neuroscience : the official journal of the Society for Neuroscience 19:19591964.

Paxinos G, Franklin KBJ (2001) The mouse brain in stereotaxic coordinates. San Diego: Elsevier Academic Press. 
Rahim RS, Meedeniya AC, Crane DI (2014) Central serotonergic neuron deficiency in a mouse model of Zellweger syndrome. Neuroscience 274:229-241.

Rambold AS, Kostelecky B, Elia N, Lippincott-Schwartz J (2011) Tubular network formation protects mitochondria from autophagosomal degradation during nutrient starvation. Proceedings of the National Academy of Sciences of the United States of America 108:10190-10195.

Robinson KM, Janes MS, Beckman JS (2008) The selective detection of mitochondrial superoxide by live cell imaging. Nature protocols 3:941-947.

Schrader M (2006) Shared components of mitochondrial and peroxisomal division. Biochim Biophys Acta 1763:531-541.

Sheng ZH, Cai Q (2012) Mitochondrial transport in neurons: impact on synaptic homeostasis and neurodegeneration. Nature reviews Neuroscience 13:77-93.

Shimozawa N, Suzuki Y, Zhang Z, Imamura A, Toyama R, Mukai S, Fujiki Y, Tsukamoto T, Osumi T, Orii T, Wanders RJ, Kondo N (1999) Nonsense and temperature-sensitive mutations in PEX13 are the cause of complementation group $\mathrm{H}$ of peroxisome biogenesis disorders. Hum Mol Genet 8:1077-1083.

Smiley ST, Reers M, Mottola-Hartshorn C, Lin M, Chen A, Smith TW, Steele GD, Jr., Chen LB (1991) Intracellular heterogeneity in mitochondrial membrane potentials revealed by a J-aggregate-forming lipophilic cation JC-1. Proceedings of the National Academy of Sciences of the United States of America 88:3671-3675.

Stamer K, Vogel R, Thies E, Mandelkow E, Mandelkow EM (2002) Tau blocks traffic of organelles, neurofilaments, and APP vesicles in neurons and enhances oxidative stress. The Journal of cell biology 156:1051-1063.

Uchida K, Stadtman ER (1992) Modification of histidine residues in proteins by reaction with 4-hydroxynonenal. Proceedings of the National Academy of Sciences of the United States of America 89:4544-4548.

Walton PA, Pizzitelli M (2012) Effects of peroxisomal catalase inhibition on mitochondrial function. Frontiers in physiology 3:108.

Wang B, Van Veldhoven PP, Brees C, Rubio N, Nordgren M, Apanasets O, Kunze M, Baes M, Agostinis P, Fransen M (2013) Mitochondria are targets for peroxisome-derived oxidative stress in cultured mammalian cells. Free radical biology \& medicine 65:882894.

Youle RJ, van der Bliek AM (2012) Mitochondrial fission, fusion, and stress. Science 337:1062-1065. 